\title{
Rates of Distress Vocalizations in Naive Domestic Chicks as an Index of Approach Tendency to an Imprinting Stimulus
}

\author{
D. W. RAJECKI, HOWARD EICHENBAUM, \\ and MARTIN HEILWEIL ${ }^{1}$ \\ Department of Psychology, The University of Michigan, \\ Ann Arbor, Michigan 48104
}

\begin{abstract}
Naive chicks were exposed to an imprinting target, consisting of a bank of miniature flashing lights, under normal $\left(100^{\circ} \mathrm{F}\right)$ and reduced temperatures $\left(75\right.$ and $57^{\circ} \mathrm{F}$ ). It was found that cold reliably evoked distress calls and that the cyclic presentations of the target substantially reduced distress calling when the target was present, relative to when it was absent. When later tested for approach tendencies to the target, clear differences between temperature groups did not emerge. Evidence was obtained, however, that chicks that showed the greatest reduction in distress calling in response to the presentation of the target under the cold conditions showed significantly greater approach tendencies in the subsequent tests.
\end{abstract}

\section{INTRODUCTION}

Tests of social attachment in imprinting research usually involve (i) a period of exposure to the target during which, presumably, the attachment develops and (ii) subsequent tests in which the strength of attachment is assessed. One test of attachment is the observation of the kind of vocalization emitted by the subject in the company of, or when separated from, the more or less familiar target. At least two kinds of vocalizations are recognized as characteristic of young precocial birds: the "contentment" and the "distress" calls. The distress calls are emitted under conditions seen to be uncomfortable (hunger, cold, pain) while the absence of distress or the presence of contentment calls occurs when conditions appear comfortable. (warm, in the presence of food) (Collias, 1952; Collias \& Joos, 1953; Driver, 1960; Guyomarc'h, 1962; Rajecki, 1972).

It is generally found that separating a young bird from an imprinting target or companion is a sufficient condition to induce distress calling (Kaufman \& Hinde, 1961). Conversely, the return of the companion, or

${ }^{1}$ This research was supported by the National Science Foundation Grant GS 3119 to Robert B. Zajonc. The authors are indebted to Robert B. Zajone for his critical comments on this paper and to Mr. E. M. Hoyt for his valuable technical assistance. Requests for reprints should be sent to D. W. Rajecki, Department of Psychology, University of Wisconsin, Madison, Wisconsin 53706. 
familiar stimulus, reduces distress calling (Zajonc, Markus, \& Wilson ${ }^{2}$ ) even when the prior exposure to the stimulus occurred prenatally (Rajecki, 1972). In a series of studies, Hoffman (Hoffman, Barrett, Ratner, \& Singer, 1972; Hoffman, Eiserer, \& Singer, 1972; Hoffman, Ratner, \& Eiserer, 1972) employed the distress calling of ducklings to investigate, among other things, the changes in behavioral control exerted by different stimuli and differing amounts of experience with those stimuli. In all these studies, vocalization behaviors are taken as an index of attachment to the familiar.

A few studies, on the other hand, have employed measures of distress calling as an index of attraction (or rejection) during initial exposure of a bird to an imprinting target. But in these, the connection between vocalizations and degree of attachment is somewhat less clear. Salzen and Tomlin (1963) found that naive chicks removed from an incubator and placed in a $75^{\circ} \mathrm{F}$ runway invariably began giving distress calls, but report only that distress ceased and was replaced by contentment calls when the chicks began to follow the target or when the target happened to touch them. Fischer (1970) reported that exposure to an imprinting target under conditions colder than ambient temperature of the rearing situation enhanced the tendency in chicks to follow. She also reported that the emission of distress calls increased as temperature decreased, and that fewer distress calls were emitted in the presence of an auditory signal than in its absence. Hess and Schaefer (1959) found that there is a direct negative relationship between the proportion of chicks in independent groups that give distress calls in an imprinting situation and the proportion of chicks likely to move toward the target during first exposure, as a function of age. However, the emission of distress calls by chicks in the presence of a novel imprinting target, or even an initial tendency to avoid it, does not necessarily preclude later approach to the same target (Bateson, 1964). Finally, Hoffman, Stratton, Newby, and Barrett (1970) showed that certain stimuli could suppress distress calling in ducklings on initial presentation. Their study does not contain a report of the degree of attachment relative to the degree of suppression, however, apparently because suppression was virtually complete under their conditions.

Since the observation of distress calling has proved such a useful tool in measuring existing attachments, it appeared profitable to employ the measurement in assessing the formation of attachment. The current study has features that allow the examination of the proposition that vocalization patterns during first exposure of a naive bird to a target are indicative of the degree of attachment resulting from that exposure.

\section{METHODS}

In a pretest, two White Leghorn chicks between 2 and $17 \mathrm{hr}$ of age were removed from a light-proof, $100^{\circ} \mathrm{F}$ incubator and placed singly in a half-inch

${ }^{2}$ Zajonc, R. B., Markus, H., and Wilson, W. R. Exposure, Object Preference, and Distress in the Domestic Chick, under editorial review. 
of ice water $\left(33-34^{\circ} \mathrm{F}\right)$ in a light-proof container for $30 \mathrm{sec}$. The animals' vocalizations were recorded on standard magnetic tape with a Panasonic RS $760 \mathrm{~S}$ recorder and an Ampex 702 microphone. Immediately after the 30-sec exposure to the cold, each animal was removed to an identical container in which the temperature was $100^{\circ} \mathrm{F}$ and vocalizations were again recorded. Durations of a sample of 30 calls from each bird (15 from each temperature condition) were measured with the aid of a storage oscilloscope. It was found that the calls emitted during the chilling ranged from 90 to $160 \mathrm{msec}$ with a mean of $114.7 \mathrm{msec}$, while the calls emitted after the birds were returned to warmth ranged from 25 to $75 \mathrm{msec}$ with a mean of $49.17 \mathrm{msec}$. These measures of the durations of the calls stemming from the various conditions substantiate the approximations of earlier researchers. Bateson (1964) estimated the length of the contentment call simply as less than one-tenth of a second and the distress call as simply greater than one-tenth of a second based on the spectrographic prints of such calls obtained by Collias and Joos (1953). Figure 1 presents photographs of examples of the two types of calls from chicks used in the pretest.

Thus distress calls were defined as those vocalizations at least $90 \mathrm{msec}$ in duration and a voice key was constructed that counted signals only that long or longer. The tapes of the pretest chicks were coded using the key and it was found that the $33-34^{\circ} \mathrm{F}$ condition elicited 89 distress calls on the average and that 14 distress calls were emitted after return of a $100^{\circ} \mathrm{F}$ setting. Hereafter "distress calls" ' refers to those vocalizations detected by the voice key.

Fertile White Leghorn eggs obtained from a commercial hatchery were held at $32^{\circ} \mathrm{F}$ for $72 \mathrm{hr}$ in order to destroy advanced embryos, thereby equating developmental age in the sample (after Gottlieb, 1963). Individual eggs were incubated, and chicks hatched and reared in inverted $16 \mathrm{oz}$ waxed cups with half-inch hardware cloth floors. The room in which the incubator was housed was darkened and chicks were handled with the aid of a penlight. Thirty birds were assigned to each of three experimental conditions and were first exposed to experimental treatment at 2-17 hr after hatching.

Subjects were removed singly from the hatching unit and transported in the wax cups to one of three identical (except for temperature) exposure units: insulated aluminum ice chests equipped with heating and cooling components. The chicks were there placed under inverted $1000 \mathrm{ml}$ translucent Pyrex beakers. The Ampex microphone was suspended next to the subject through an opening in the base of the beaker. The imprinting target was a bank of five miniature bulbs (28 V, $40 \mathrm{~mA}$ ) covered with Sylvania 81001-0 translucent red caps that were fixed to the outer surface of the beaker a half-inch apart along a vertical line. When activated, the bulbs flashed in a sequence from uppermost down. A sequence was completed in approximately $1 / 2 \mathrm{sec}$ with a 2 -sec interval between sequences.

Each chick was kept in an exposure unit for $10 \mathrm{~min}$. During that period the target array was alternately deactivated and activated at 1-min intervals. 


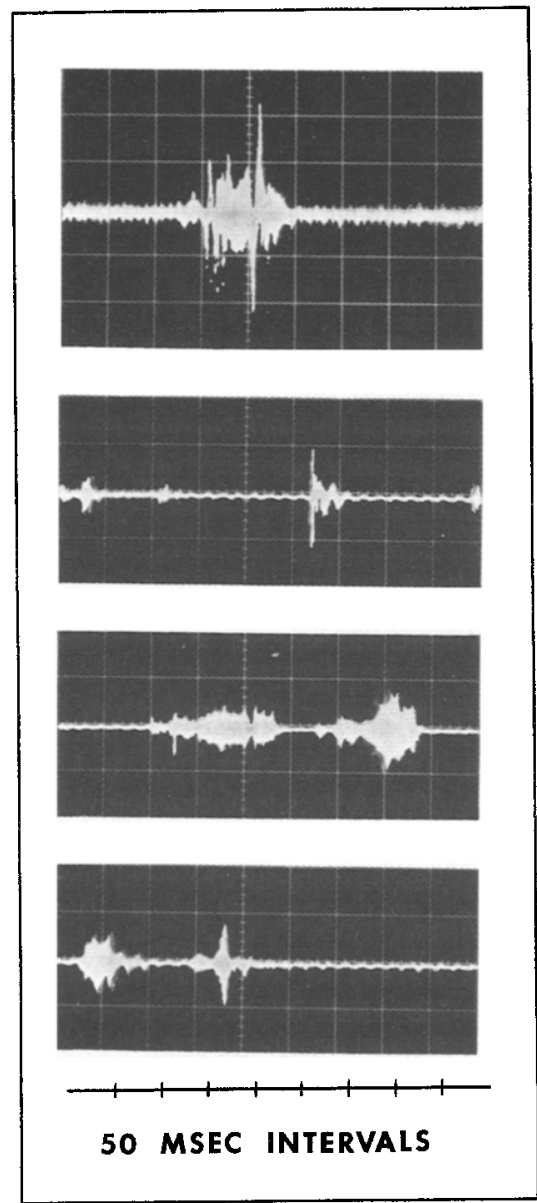

CHICK 1

DISTRESS

\section{CHICK 1 \\ CONTENTMENT}

\section{CHICK 2 \\ DISTRESS}

\section{CHICK 2}

CONTENTMENT

Fig. 1. Storage oscilloscope photographs of representative distress (mean duration $=114.7 \mathrm{msec}$ ) and contentment (mean duration $=49.17 \mathrm{msec}$ ) vocalizations from pretest subjects.

Continuous recordings of the chicks' vocalizations throughout this period were made with an Ampex 1100 recorder. Groups of 30 chicks were exposed under one of three experimental temperature conditions. One group of chicks was exposed at $100^{\circ} \mathrm{F}$, the temperature at which all subjects were incubated, hatched, and reared to the time of treatment. A second group was exposed in an exposure unit at room temperature, about $75^{\circ} \mathrm{F}$. For the final group's exposure, the surface temperature of the floor of one of the exposure units was maintained at approximately $57^{\circ} \mathrm{F}$ by the installation of ice-filled containers. Chicks were assigned to these conditions through a schedule by 
which assignment to a given condition temporally preceeded and followed assignment to any other condition equally.

After the end of the exposure session all chicks were tested for tendency to approach the imprinting target. Half the subjects were tested immediately after exposure and half were returned to the incubator inside a wax cup for $1 \mathrm{hr}$ prior to testing. The testing situation was a $7 \times 7 \times 48$ in. alley placed in a sound-treated box and dimly illuminated by two General Electric D8 lamps. The temperature in the alley was approximately $75^{\circ} \mathrm{F}$. Identical targets were installed at both ends of the alley and could be activated alternately by a switch. A subject was placed in the center of the alley with the target activated at one end. The bird's position was noted every $10 \mathrm{sec}$ for a period of nine 1-min blocks. If the chick approached to within $12 \mathrm{in.} \mathrm{of} \mathrm{the} \mathrm{target} \mathrm{(when} \mathrm{activated)} \mathrm{within} \mathrm{any} \mathrm{given} \mathrm{minute,} \mathrm{the} \mathrm{array} \mathrm{was}$ deactivated at the end of that minute at that end of the runway. The target was simultaneously activated at the opposite end. The scores assigned were the distances the subjects "followed" the target, in inches.

\section{RESULTS}

The vocalization patterns emitted by the birds under the various exposure temperatures are quite striking. Inspection of Fig. 2 shows substantial overall differences between the three treatment groups

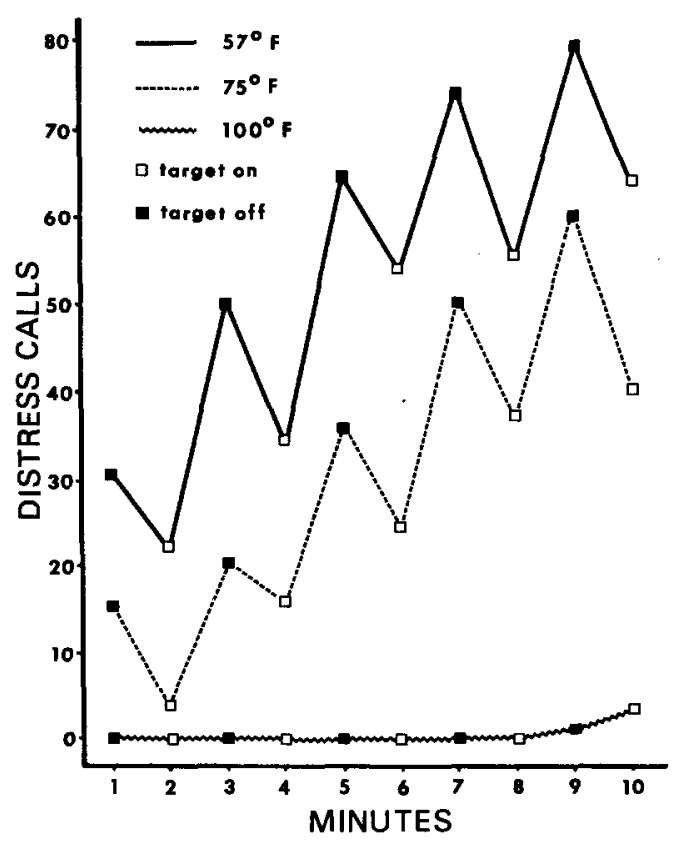

Fig. 2. Average number of distress calls as a function of temperature, time, and the absence or presence of the imprinting target during initial exposure. 
$(F(2,87)=48.52, P<.01)$ and also that there are striking reductions of calls when the target was activated $(F(1,783)=53.75, P<.01)$. Time was a significant factor $(F(9,783)=43.45, P<.01)$ with more calls later than earlier in the exposure session, presumably due to a drop in the subjects' own temperatures. Finally, the effects of time and the activation of the target had differential effects for the birds exposed at the different temperatures. In general, the lower the temperature the greater the effect as shown in the Temperature $X$ Time $(F(18,783)=10.81, P<.01)$ and the Temperature $X$ Target Activation $(F(2,783)=15.08, P<.01)$ interactions.

As a supplementary index that the cold conditions were indeed stressful, the subjects that defecated were noted. Only 18 of the 90 birds exposed did so, but defecation was clearly related to temperature: 1 instance in the $100^{\circ} \mathrm{F}$ condition, 5 in the $75^{\circ} \mathrm{F}$ condition, and 12 in the $57^{\circ} \mathrm{F}$ condition $\left(\mathrm{x}^{2}(2)=10.34, P<.01\right)$.

The test for approach tendency after exposure showed that the intermediate temperature $\left(75^{\circ} \mathrm{F}\right)$ produced the most following (see Fig. 3) but these differences were not significant $(F<1)$. Significant, however, are the differences between birds tested with a 0 -hr delay and those tested with a 1-hr delay after exposure $(F(1,84)=5.46, P<.05)$ with the latter proving to be the better followers.

Because it was possible to identify subjects individually, a post hoc analysis of following related to vocalization patterns in the $75^{\circ} \mathrm{F}$ and the $57^{\circ} \mathrm{F}$ groups was undertaken. During initial exposure some birds in these groups emitted the patterns seen in Fig. 2 more than others. Whether a bird

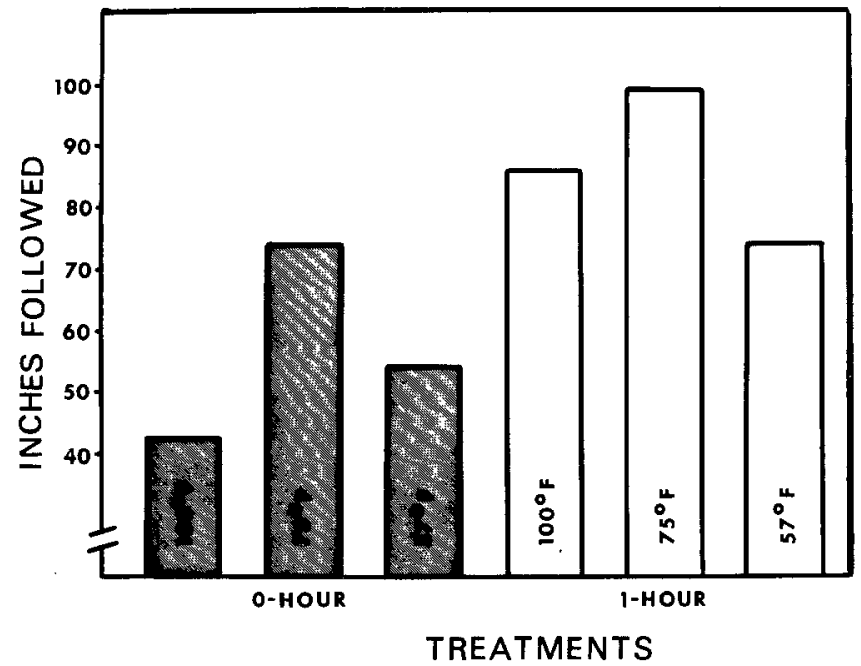

Fig. 3. Average distance in inches of following the imprinting target by chicks tested immediately, or with a 1-hr delay after the initial exposure. 
exhibited this pattern or not was determined by subtracting its total calls when the target was activated from its total calls when deactivated. The subjects in each group were then rank-ordered on the basis of this difference ("D") and the approach tendency of the birds in the first 15 ranks (high "D") was compared to that of the last 15 (low "D"). These differences are shown in Fig. 4 where it can be seen that responsiveness (in terms of suppression of distress calls when the target was activated) during initial exposure is related to later approach behavior $(F(1,56)=5.03, P<.05)$. Neither the differences between temperature groups $\left(75 \mathrm{vs} 57^{\circ}\right)$ nor the " $D$ " $X$ Temperature interaction were significant, however. Therefore, it can be said that the birds whose rates of distress calling were most affected by the presentation of the target proved to be the best "followers."

It was somewhat disappointing that the birds in the $100^{\circ} \mathrm{F}$ group emitted so few distress calls. In fact, only 6 of the 30 emitted any at all. It was possible to rank-order these few subjects on the " $D$ " dimension, though, and compare their later approach behavior. For the 3 high " $D$ ", $100^{\circ}$ F-treated animals, the average approach score was 68 in. and for the low " $D$ " counterparts the mean was 43 in. While this difference is in a direction consistent with the findings for the other temperature groups, the sample here is too small to yield statistical significance.

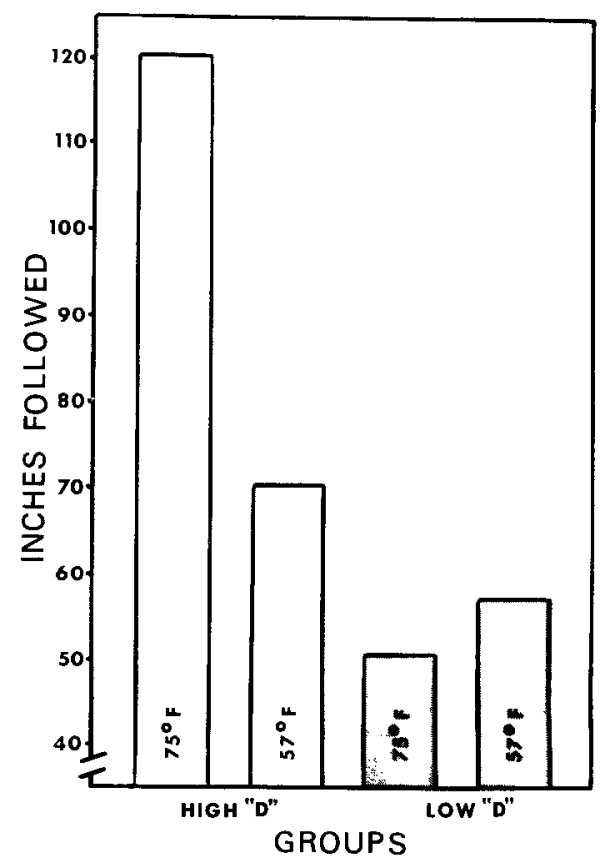

Fig. 4. Average distance in inches of following the imprinting target by chicks defined as high- or low-"D" (see text) in the $75^{\circ}$ F- and the $57^{\circ}$ F-groups. 


\section{DISCUSSION}

The findings of this experiment indicate that there is a reduction in the number of distress calls from naive chicks due to the presentation of an imprinting stimulus. They are in line with the conclusion of Hoffman et al. (1970) that such a stimulus exercises an immediate control over certain behaviors in young precocial birds.,

Clear evidence was not obtained for the relationship between the effect of cold during exposure and subsequent approach behavior. This lack of effect is inconsistent with Fischer's (1970) findings. One would be wise to examine procedural differences between the two studies, of course, but it may simply be that a total of $5 \mathrm{~min}$ of exposure to a particular target, as in the current work, is not a sufficient period for general treatment effects to obtain. This is probably the case as it is known that imprinting is not an instantaneous or an all-or-none process. Rather, attachments are more likely to form with prolonged or repeated exposure to a target (Hoffman, Eiserer, \& Singer, 1972; Saegert \& Rajecki, 1973; Zajonc, Reimer, \& Hausser, in press).

Still, it may be of some theoretical significance that even the chicks that evinced considerable distress (witness also the defecation data) showed a substantial amount of following later. This was also the case with chicks receiving the heaviest shock applications in a study by Kovach and Hess (1963). These sorts of findings seem to be in contrast with the position that imprinting (or the emergence of approach and following) is interpretable in terms of simple associative learning (for a review of interpretations see Rajecki, 1973).

The difference in birds tested immediately or at a later time are consistent with the findings of Sluckin and Taylor (1964) that responsiveness is positively related to length of delay between exposure and testing, presumably up to a point. This difference cannot be attributed to motor capacity as influenced by cold because it is consistent across all groups.

The difference between subjects tested on the basis of high and low " $D$ "-scores is one of the most interesting findings of this study. That this difference exists indicates that vocalization patterns during exposure are indeed related to subsequent approach tendencies and lends weight to the work of Hoffman et al. (1970).

\section{REFERENCES}

Bateson, P. P. G. (1964) Changes in chicks' responses to novel moving objects over the sensitive period for imprinting. Anim. Behav. 12, 479-489.

Collias, N. E. (1952) The development of social behavior in birds. Auk 69, 127-159.

Collias, N. E. \& Joos, M. (1953) The spectrographic analysis of sound signals of the domestic fowl. Behaviour 5, 175-187. 
Driver, P. M. (1960) Field studies on the behaviour of sea-ducklings. Arctic 13, 201-204.

Fischer, G. J. (1970) Arousal and impairment: Temperature effects on following during imprinting. J. Comp. Physiol. Psychol. 73, 412-420.

Gottlieb, G. (1963) Refrigerating eggs prior to incubation as a way of reducing error in calculating developmental age in imprinting experiments. Anim. Behav. 11, 290-292.

Guyomarc'h. J. -Ch. (1962) Contribution a l'etude du comportement vocal du poussin de Gallus Domesticus. J. Psychol. Norm. Pathol. 3, 283-306.

Hess, E. H. \& Schaefer, H. H. (1959) Innate behavior patterns as indicators of the "critical period." Z. Tierpsychol. 16, 155-160.

Hoffman, H. S., Barrett, J., Ratner, A. \& Singer, D. (1972) Conditioned suppression of distress calls in imprinted ducklings. J. Comp. Physiol. Psychol. 80, 357-364.

Hoffman, H. S., Eiserer, L. A. \& Singer, D. (1972) Acquisition of behavioral control by a stationary imprinting stimulus. Psychon. Sci. 26, 146-148.

Hoffman, H. S., Ratner, A. M. \& Eiserer, L. A. (1972) Role of visual imprinting in the emergence of specific filial attachments in ducklings. J. Comp. Physiol. Psychol. 81, 399-409.

Hoffman, H. S., Stratton, J. W., Newby, V. \& Barrett, J. E. (1970) Development of behavioral control by an imprinting stimulus. J. Comp. Physiol. Psychol. 71, 229-236.

Kaufman, J. C. \& Hinde, R. A. (1961) Factors influencing distress calling in chicks, with special reference to temperature and social isolation. Anim. Behav. 9, 197-204.

Kovach, J. K. \& Hess, E. H. (1963) Imprinting: Effects of painful stimulation upon the following response. J. Comp. Physiol. Psychol. 56, 461-464.

Rajecki, D. W. (1972) Effects of prenatal exposure to auditory and visual stimuli on social responses in chicks. Doctoral Dissertation, University of Michigan.

Rajecki, D. W. (1973) Imprinting in precocial birds: Interpretation, evidence and evaluation. Psych. Bull. 79, 48-58.

Saegert, S. \& Rajecki, D. W. (1973) Effects of prior exposure to animate objects on approach tendency in chicks. Behav. Biol. 8, 749-754.

Salzen, E. A. \& Tomlin, F. J. (1963) The effect of cold on the following response of the domestic fowl. Anim. Behav. 11, 62-65.

Sluckin, W. \& Taylor, K. F. (1964) Imprinting and short-term retention. Brit. J. Psychol, 55, 181-187.

Zajonc, R. B., Reimer, D. J. \& Hausser, D. (1973) Imprinting and the development of object preference in chicks by mere repeated exposure. J. Comp. Physiol. Psychol, in press. 